\title{
A GENERALIZATION OF ROLLE'S THEOREM AND AN APPLICATION TO A NONLINEAR EQUATION
}

\author{
ANTONIO TINEO
}

(Received 10 November 1986)

Communicated by A. J. Pryde

\begin{abstract}
Given two $C^{1}$-functions $g: \mathbf{R} \rightarrow \mathbf{R}, u:[0,1] \rightarrow \mathbf{R}$ such that $u(0)=u(1)=0, g(0)=0$, we prove that there exists $c$, with $0<c<1$, such that $u^{\prime}(c)=g(u(c))$. This result implies the classical Rolle's Theorem when $g \equiv 0$. Next we apply our result to prove the existence of solutions of the Dirichlet problem for the equation $x^{\prime \prime}=f\left(t, x, x^{\prime}\right)$.
\end{abstract}

1980 Mathematics subject classification (Amer. Math. Soc.): 34 B 15.

\section{Introduction}

Let $f:[0,1] \times \mathbf{R} \times \mathbf{R} \rightarrow \mathbf{R}$ be a continuous function and suppose that there exist a continuous function $\phi:[0, \infty) \rightarrow(0, \infty)$ and a constant $R>0$ such that

$$
\begin{gathered}
f(t, x, 0) x \geq 0 \quad \text { if }|x|=R, \\
|f(t, x, y)| \leq \phi(|y|) \quad \text { if }|x| \leq R .
\end{gathered}
$$

It is well known that

\subsection{THEOREM. The Dirichlet problem}

$$
x^{\prime \prime}=f\left(t, x, x^{\prime}\right), \quad x(0)=x(1)=0
$$

(C) 1989 Australian Mathematical Society 0263-6115/89 \$A2.00+0.00 
has at least one solution if

$$
\int_{0}^{\infty} s \phi(s)^{-1} d s>R
$$

For instance see [1] or [2].

In this paper we prove a generalized Rolle's Theorem and we apply this result to obtain the following generalization of Theorem 0.1 .

0.2. TheOREM. Suppose that there are $\left(r_{0}, s_{0}\right),\left(r_{1}, s_{1}\right) \in \mathbf{R} \times \mathbf{R}, r_{1}<0<r_{0}$, such that

(i) $f\left(t, x, s_{0}\right) \geq 0$ if $r_{0} \leq x \leq r_{0} \exp (K)$,

(ii) $f\left(t, x, s_{1}\right)<0$ if $r_{1} \exp (K) \leq x \leq r_{1}$, where $K=\max \left\{\left|s_{0} / r_{0}\right|,\left|s_{1} / r_{1}\right|\right\}$. Assume further that

(iii) $|f(t, x, y)| \leq \phi(|y|)$ if $r_{1} \exp (K) \leq x \leq r_{0} \exp (K)$,

(iv) $\int_{0}^{\infty} s \phi(s)^{-1} d s>\max \left\{-r_{1}, r_{0}\right\} \exp (K)$.

Then the problem $(0.1)$ has at least one solution $v$ such that $r_{1} \exp (K) \leq v \leq r_{0}$ $\exp (K)$.

\section{A general existence principle}

In the following, $C_{0}^{2}$ denotes the space of functions $u:[0,1] \rightarrow \mathbf{R}$ of class $C^{2}$ such that $u(0)=u(1)=0$, with the usual norm $\|u\|_{2}=\max \left\{\left\|u^{(i)}\right\|_{0}, i=\right.$ $0,1,2\}$, where $\left\|u^{(i)}\right\|_{0}=\sup \left\{\left|u^{(i)}(t)\right|: 0 \leq t \leq 1\right\}$. For reference purposes, we state the following general, and now classical, result (see [2] for details).

1.1. THEOREM. Let $U$ be an open and bounded neighborhood of $0 \in C_{0}^{2}$ such that the problem

$$
x^{\prime \prime}=\lambda f\left(t, x, x^{\prime}\right), \quad x(0)=x(1)=0
$$

has no solutions in the boundary $\partial U$ of $U$ for $0<\lambda<1$. Then the problem $(0.1)$ has at least one solution in the closure $\mathrm{cl}(U)$ of $U$.

\section{A Nagumo inequality}

In this section we obtain a priori bounds for derivatives: 
2.1. Proposition. Let $v \in C_{0}^{2}$. If $v^{\prime}\left(t_{0}\right) \neq 0$ then there is an interval $[a, b] \subset[0,1]$ such that $v$ and $v^{\prime}$ have constant sign in $(a, b) ; t_{0} \in\{a, b\}$ and $v^{\prime}$ has a zero at one of the endpoints of $[a, b]$.

Proof. We consider two cases.

Case $1 ; v\left(t_{0}\right) \neq 0$. Since $v(0)=v(1)=0$ there is an interval $[c, d] \subset[0,1]$ such that $v(t) \neq 0$ if $t \in(c, d), v(c)=v(d)=0$ and $c<t_{0}<d$. In particular $v^{\prime}\left(t_{1}\right)=0$ for some $t_{1} \in[c, d]$ and hence there is an interval $[a, b] \subset[c, d]$ such that $t_{0} \in\{a, b\}, v^{\prime}(a) \cdot v^{\prime}(b)=0$ and $v^{\prime}(t) \neq 0$ for $t \in(a, b)$, as required.

Case 2; $v\left(t_{0}\right)=0$. Since $v^{\prime}\left(t_{0}\right) \neq 0$ there is an interval $[c, d] \subset[0,1]$ such that $t_{0} \in\{c, d\}, v(c)=v(d)=0$ and $v(t) \neq 0$ if $t \in(c, d)$. The proof follows as in the first case.

2.2. Corollary. Let $\phi:[0, \infty) \rightarrow(0, \infty)$ be a continuous function and let $v \in C_{0}^{2}$ be such that $\left|v^{\prime \prime}(t)\right| \leq \phi\left(\left|v^{\prime}(t)\right|\right)(0 \leq t \leq 1)$. Then

$$
\int_{0}^{\left|v^{\prime}(t)\right|} s \phi(s)^{-1} d s \leq\|v\|_{0} \quad(0 \leq t \leq 1) .
$$

Proof. Let $t_{0} \in[0,1]$ be such that $v^{\prime}\left(t_{0}\right) \neq 0$ and take $[a, b] \subset[0,1]$ as given by Proposition 2.1. If we follow the proof of Theorem 3.1 of [2] then we get

$$
\int_{0}^{\left|v^{\prime}\left(t_{0}\right)\right|} s \phi(s)^{-1} d s \leq|v(a)-v(b)|,
$$

so the proof is complete, since $v$ has constant sign in $(a, b)$.

\section{A generalized Rolle's Theorem}

From now on $h: \mathbf{R} \rightarrow \mathbf{R}$ denotes a function of class $C^{1}$. Given $u \in C_{0}^{2}$ and $a \in[0,1]$ we define

$$
\begin{gathered}
u_{a}(t)=u(t) \exp \left(-\int_{a}^{t} h(u(s)) d s\right), \\
M(u)=\left\{a \in[0,1]: \max u_{a}=u(a)>0\right\} \\
m(u)=\left\{a \in[0,1]: \min u_{a}=u(a)<0\right\}
\end{gathered}
$$

3.1. LeMMA. If $\max u>0$ (respectively $\min a<0$ ) then $M(u)$ (respectively $m(u))$ is a nonempty set. 
Proof. If $\max u>0$ we get $\max u_{0}=u_{0}(a)>0$ for some $a \in[0,1]$. On the other hand $u_{a}=k u_{0}$ for some $k>0$ and hence $0<\max u_{a}=k \max u_{0}=$ $k u_{0}(a)=u_{a}(a)=u(a)$; or $a \in M(u)$. Similarly $m(u) \neq \varnothing$ if $\min u<0$.

3.2. Remarks. (a) If $a \in M(u)$ one has $u_{a}^{\prime}(a)=0$ and $u_{a}^{\prime \prime}(a) \leq 0$, which is equivalent to

$$
u^{\prime}(a)=u(a) h(u(a))
$$

and

$$
u^{\prime \prime}(a) \leq u^{\prime}(a) \cdot\left[h(u(a))+u(a) h^{\prime}(u(a))\right] .
$$

(b) If $a \in m(u)$ we obtain (3.2) and the reverse of inequality (3.3).

Notice that $\max u_{a}=u_{a}(a)$ (respectively $\min u_{a}=u_{a}(a)$ ) if $a \in M(u)$ (respectively $a \in m(u)$ ).

REMARK. Let $u:[0,1] \rightarrow \mathbf{R}$ a differentiable function and define $u_{a}$ by (3.1) for $a \in[0,1]$. If $u(0)=u(1)=0$ we get $u_{a}(0)=u_{a}(1)=0$ and hence $u_{a}^{\prime}(c)=0$ for some $c \in(0,1)$. Therefore $u^{\prime}(c)=u(c) h(u(c))$. This result implies Rolle's Theorem when $h \equiv 0$.

For each $r>0$ let

$$
\begin{aligned}
& U(r)=\left\{u \in C_{0}^{2}: M(u) \neq \varnothing, u_{a}(t)<r \text { if }(a, t) \in M(u) \times[0,1]\right\}, \\
& V(-r)=\left\{u \in C_{0}^{2}: m(u) \neq \varnothing, u_{a}(t)>r \text { if }(a, t) \in m(u) \times[0,1]\right\}, \\
& U(r, 0)=U(r) \cup U(0), V(-r, 0)=V(-r) \cup V(0),
\end{aligned}
$$

where $U(0)=\left\{u \in C_{0}^{2}: M(u)=\varnothing\right\}$ and $V(0)=\left\{u \in C_{0}^{2}: m(u)=\varnothing\right\}$.

We give now some properties of the sets $U(r, 0), v(-r, 0)$, that we shall use in the next section.

3.3. Proposition. (a) If $u \notin U(r, 0)$ and $u \in C_{0}^{2}$ (respectively $u \notin V(-r, 0)$ ) then there is $a \in M(u)$ (respectively $a \in m(u)$ ) such that $u(a) \geq r$ (respectively $u(a) \leq-r)$.

(b) $U(r, 0), V(-r, 0)$ are open sets $(r>0)$.

(c) $\partial\left(U\left(r_{0}, 0\right) \cap V\left(r_{1}, 0\right)\right) \subseteq\left(\partial U\left(r_{0}, 0\right)\right) \cup\left(\partial V\left(r_{1}, 0\right)\right), r_{1}<0<r_{0}$.

(d) $I f|h(x)| \leq K$ for all $x \in \mathbf{R}$ (some $K \geq 0)$ and $u \in \operatorname{cl}\left(U\left(r_{0}, 0\right) \cap V\left(r_{1}, 0\right)\right.$ ) for some $r_{1}<0<r_{0}$, then $r_{1} \exp (K) \leq u(t) \leq r_{0} \exp (K)(0 \leq t \leq 1)$.

Proof. (a) This is trivial.

(b) Let $\left\{u_{n}\right\}$ be a sequence in $C_{0}^{2}$ which tends to $u \in C_{0}^{2}$ in the \|\|$_{2}$-norm; then $\left\{u_{n, a_{n}}\right\}$ converges uniformly to $u_{a}$ if $a_{n} \rightarrow a$. Since $[0,1]$ is a compact set it is not difficult to prove that the complement of $U(r, 0)$ (respectively $V(r, 0))$ is a closed set.

(c) This is a consequence of (b). 
Finally, to prove (d), notice first that $U\left(r_{0}, 0\right) \cap V\left(r_{1}, 0\right)$ is the union of the sets $U\left(r_{0}\right) \cap V\left(r_{1}\right), U\left(r_{0}\right) \cap V(0), V\left(r_{1}\right) \cap U(0)$ and $U(0) \cap V(0)$. Secondly, by Lemma 3.1, $U(0)=\left\{u \in C_{0}^{2}: u \leq 0\right\}$ and $V(0)=\left\{u \in C_{0}^{2}: u \geq 0\right\}$. If $u \neq 0$ it is easy to prove that one has the following cases: (i) there are $a, b \in[0,1]$ such that $\max u_{a} \leq r_{0}$ and $\min u_{b} \geq r_{1}$; (ii) $u \geq 0$ and $\max u_{a} \leq r_{0}$ for some $a \in[0,1]$;

(iii) $u \leq 0$ and $\min u_{b} \geq r_{1}$ for some $b \in[0,1]$.

The proof follows from the fact that

$$
u(t)=u_{a}(t) \exp \left(\int_{a}^{t} h(u(s)) d s\right) \quad \text { for } a, t \in[0,1]
$$

\section{The proof of Theorem 0.2}

Let $\rho, \varepsilon_{0}>0$ be such that

$$
\int_{0}^{\rho} \frac{s d s}{\phi(s)+\varepsilon_{0}}>\max \left\{-r_{1}, r_{0}\right\} \exp (K)
$$

For some $\varepsilon_{1}>0$ one has

$$
\int_{0}^{\rho} \frac{s d s}{\phi(s)+\varepsilon_{0}}=\max \left\{-r_{1}, r_{0}\right\} \exp \left(K+\varepsilon_{1}\right)
$$

Claim. If there is $\varepsilon \in\left(0, \varepsilon_{1}\right)$ such that

$$
|f(t, x, y)| \leq \phi(|y|) \text { for } r_{1} \exp (K+\varepsilon) \leq x \leq r_{0} \exp (K+\varepsilon)
$$

then the problem $(0.1)$ has at least one solution $V$ such that $r_{1} \exp (K) \leq$ $v(t) \leq r_{0} \exp (K)$.

Proof of the claim. By the Tietze-Uryshon Lemma there is a continuous functin $\Delta: \mathbf{R} \times \mathbf{R} \rightarrow[-1,1]$ such that $\Delta\left(x, s_{0}\right)=1$ if $r_{0} \leq x \leq r_{0} \exp (k)$, and $\Delta\left(x, s_{1}\right)=-1$ if $r_{1} \exp (K) \leq x \leq r_{1}$.

For each integer $n$ such that $n \varepsilon_{0} \geq 1$, we let $f_{n}(t, x, y)=f(t, x, y)+$ $n^{-1} \Delta(x, y)$. Now fix $n$ with $n \varepsilon_{0} \geq 1$, and notice that there is $\delta=\delta_{n}>0$ with $\delta \leq \min \{\varepsilon, 1 / n\}$ such that

$$
\begin{array}{ll}
f_{n}\left(t, x, s_{0}\right)>0 & \text { if } r_{0} \leq x \leq r_{0} \exp (K+\delta), \\
f_{n}\left(t, x, s_{1}\right)<0 & \text { if } r_{1} \exp (K+\delta) \leq x \leq r_{1} .
\end{array}
$$

Choose a $C^{1}$-function $h=h_{n}: \mathbf{R} \rightarrow \mathbf{R}$ such that $h\left(r_{i}\right)=s_{i} / r_{i}, h^{\prime}\left(r_{i}\right)=$ $-s_{i} / r_{i}^{2}, h(x)=s_{0} / s$ if $x \geq r_{0}, h(x)=s_{1} / x$ if $x \leq r_{1}$, and $|h(x)| \leq K+\delta$ for $x \in \mathbf{R}$. 
Given $u \in C_{0}^{2}$ and $a \in[0,1]$ define $u_{a}$ by (3.1) and let $U$ be the open and bounded neighborhood of $0 \in C_{0}^{2}$ defined by $u \in U$ if and only if

$$
u \in U\left(r_{0}, 0\right) \cap V\left(r_{1}, 0\right), \quad\left\|u^{\prime}\right\|_{0}<\rho, \quad\left\|u^{\prime \prime}\right\|_{0}<R,
$$

where $R=R_{n}>0$ is chosen such that

$$
\left|f_{n}(t, x, y)\right|<R \text { if }|x| \leq M:=\max \left\{-r_{1}, r_{0}\right\} \exp (K+\delta),
$$

and

$$
|y| \leq \rho(0 \leq t, \lambda \leq 1) .
$$

We shall prove that the problem

$$
x^{\prime \prime}=\lambda f_{n}\left(t, x, x^{\prime}\right), \quad x(0)=x(1)=0
$$

has no solutions on $\partial U$ for $0<\lambda<1$.

Suppose that $u \in \operatorname{cl}(U)$ is a solution of $(4.6)_{\lambda}$ for some $\lambda \in(0,1)$; by Proposition 3.3(d) we obtain

$$
r_{1} \exp (K+\delta) \leq u(t) \leq r_{0} \exp (K+\delta)
$$

and by $(4.6)_{\lambda},(4.5)$ and (4.2), $\left|u^{\prime \prime}(t)\right| \leq 1 / n+\phi\left(\left|u^{\prime}(t)\right|\right)$ since $\delta \leq \varepsilon$. On the other hand, $n \varepsilon_{0} \geq 1$ and $\delta \leq \varepsilon<\varepsilon_{1}$, and therefore

$$
\int_{0}^{\rho} s\left[1 / n+\left.\phi(s)\right|^{-1} d s>\max \left\{-r_{1}, r_{0}\right\} \exp (K+\delta) \geq\|u\|_{0},\right.
$$

and by Corollary 2.2 we get $\left\|u^{\prime}\right\|_{0}<\rho$. Thus, by (4.5) and (4.6) $\lambda,\left\|u^{\prime \prime}\right\|_{0}<R$.

If $u \in \partial U$ then $u \in\left(\partial U\left(r_{0}, 0\right) \cup\left(\partial V\left(r_{1}, 0\right)\right)\right.$ and we suppose first that $u \in \partial U\left(r_{0}, 0\right)$. In this case, by Proposition 3.3(a), there is $a \in M(u)$ such that $\max u_{a}=u_{a}(a)=u(a) \geq r_{0}$ and by remarks 3.2 and the definition of $h$ we have

$$
u^{\prime}(a)=u(a) h(u(a))=s_{0}
$$

and

$$
u^{\prime \prime}(a) \leq s_{0}\left[h(u(a))+u(a) h^{\prime}(u(a))\right]=0
$$

as $h(u(a))=s_{0} / u(a)$ and $h^{\prime}(u(a))=-s_{0} / u(a)^{2}$.

But this is a contradiction since, by (4.7) and (4.3), $u^{\prime \prime}(a)=\lambda f_{n}\left(a, u(a), s_{0}\right)$ $>0$. This contradiction proves that $u \notin \partial U\left(r_{0}, 0\right)$. Analagously $u \notin \partial V\left(r_{1}, 0\right)$ and then $u \notin \partial U$. So, by Theorem 1.1, the problem (4.6) ${ }_{1}$ has at least one solution $v_{n}$ such that $\left\|v_{n}^{\prime}\right\|_{0} \leq \rho,\left\|v_{n}^{\prime \prime}\right\| \leq R$ and $r_{1} \exp (K+1 / n) \leq v_{n}(t) \leq$ $r_{0} \exp (K+1 / n)$. Remember that $\delta \leq 1 / n$. Now it is easy to prove that $\left\{v_{n}\right\}$ has a subsequence which converges in $C_{0}^{2}$ to a solution of $(0.1)$. So the proof of the claim is finished. that

Now take an arbitrary $\varepsilon \in\left(0, \varepsilon_{1}\right)$ and a continuous function $\alpha: \mathbf{R} \rightarrow \mathbf{R}$ such

$$
\begin{gathered}
\alpha(x)=x \quad \text { if } r_{1} \exp (K) \leq x \leq r_{0} \exp (K), \\
\alpha\left(\left[r_{1} \exp (K+\varepsilon), r_{0} \exp (K+\varepsilon)\right]\right) \subset\left[r_{1} \exp (K), r_{0} \exp (K)\right],
\end{gathered}
$$


and define $g(t, x, y)=f(t, \alpha(x), y)$. We have

$$
\begin{array}{cl}
g\left(t, x, s_{0}\right) \geq 0 & \text { if } r_{0} \leq x \leq r_{0} \exp (K), \\
g\left(t, x, s_{1}\right) \leq 0 & \text { if } r_{1} \exp (K) \leq x \leq r_{1}, \\
|g(t, x, y)| \leq \phi(|y|) & \text { if } r_{1} \exp (K+\varepsilon) \leq x \leq r_{0} \exp (K+\varepsilon) .
\end{array}
$$

Then, by the claim, there exists at least one solution $v$ of the problem

$$
x^{\prime \prime}=g\left(t, x, x^{\prime}\right), \quad x(0)=x(1)=0
$$

such that $r_{1} \exp (K) \leq v(t) \leq r_{0} \exp (K)$. In particular $\alpha(v(t))=v(t)(0 \leq$ $t \leq 1)$ and hence $v$ is a solution of $(0.1)$. So the Proof of Theorem 0.2 is complete.

\section{References}

[1] R. T. Graines and J. L. Mawhin, Coincidence degree and nonlinear differential equations (Lectures Notes in Math., 568, Springer-Verlag, Berlin, Heidelberg, New York, 1977).

[2] A. Granas, R. B. Guenther and J. W. Lee, 'Nonlinear boundary value problems for some class of ordinary differential equations', Rocky Mountain J. Math. 10 (1980), 35-58.

Departamento de Matematicas

Universidad de Los Andes

Facultad de Ciencias

Merida, Edo Merida

Venezuela 